\title{
Relativistic quantum defect orbital study of forbidden transitions in $\mathrm{Co}^{16+\star}$
}

\author{
E. Charro and I. Martín \\ Departamento de Química Física, Universidad de Valladolid, 47011 Valladolid, Spain
}

Received 8 June 2001 / Accepted 2 July 2001

\begin{abstract}
Theoretical line strengths and transition probabilities for electric quadrupole (E2) transitions between levels belonging to several important configurations of $\mathrm{Co}^{16+}$ are reported. The calculations have been carried out with the Relativistic Quantum Defect Orbital (RQDO) method. A general good accord between the present results and some earlier theoretical data is found. Some of the E2 transition probabilities are reported for the first time. The correct systematic behaviour of the RQDO line strengths along the spectral series is reproduced by the present results in the absence of configuration mixing. This semiempirical method appears to adequately describe E2 transitions in this medium- $Z$ atomic system.
\end{abstract}

Key words. atomic data

\section{Introduction}

Most theoretical determinations of atomic oscillator strengths have concentrated on allowed electric dipole transitions. Electric quadrupole transitions, however, are also of astrophysical interest: a number of such transitions have been observed in the ultraviolet spectrum of the solar corona; moreover, many gaseous nebulae exhibit in their spectra forbidden lines of low excitation energy belonging to alkali-like ions. The detailed study of these astronomical objects requires the knowledge of electric quadrupole transition probabilities. Additionally, the determination of electric quadrupole oscillator strengths can help in assessing the contribution of electric quadrupole radiation to multiphoton transitions.

Ionized iron, nickel and cobalt are astrophysically very important, and also as impurities in magnetic fusion plasmas (see e.g. Kurucz 1990).

The relatively high cosmic abundances of the iron group elements in stellar objects stimulates interest in these elements. Cobalt, being an odd- $Z$ number element, is far less abundant than the even- $Z$ elements of the group: $\mathrm{Cr}, \mathrm{Fe}$ and Ni. However, a number of lines of cobalt have been identified in astrophysical studies. Ultraviolet

Send offprint requests to: E. Charro,

e-mail: Imnieto@wamba.cpd.uva.es

* Table 4 is only available in electronic form at the CDS via anonymous ftp to cdsarc.u-strasbg.fr

$(130.79 .128 .5)$ or via

http://cdsweb.u-strasbg.fr/cgi-bin/qcat?J/A+A/376/1106 spectroscopy is used for impurity monitoring in controlled thermonuclear fusion. This technique has been chosen given its ability to measure lines of both light $(\mathrm{O}, \mathrm{C})$ and the usual heavy ( $\mathrm{Fe}, \mathrm{Ni}$ and $\mathrm{Co}$ ) impurities.

The results obtained in the present work follow the expected regularities along spectral series for these E2 transitions. Such systematic trend studies have proved extremely useful for analysing, evaluating and predicting large quantities of $S$ - or $f$-value data, mostly on E1 transitions (Martin \& Wiese 1996). The value of this approach lies on the fact that once the trend has been established in graph form for a particular spectral series, in a given atomic system, data for transitions involving higher levels can be estimated in a very simple way.

$\mathrm{Co}^{16+}$ belongs to the sodium isolectronic sequence, with a $[\mathrm{Ne}] 3 \mathrm{~s}$ configuration for the ground state. Only very few experimental results are available concerning electric quadrupole transition probabilities along the sodium isoelectronic sequence, and most of them refer to atomic sodium. Most of the calculations done for sodium-like systems correspond to allowed transitions. To our knowledge, there are no recent calculations or measurements of transition probabilities for forbidden lines in $\mathrm{Co}^{16+}$. Earlier data are the multiplet $S$-values calculated by Tull et al. (1972) with a Hartree-Fock procedure of frozen-core type, and a critical compilation by Fuhr et al. (1988) of transition probability data and $S$-values for forbidden lines of $\mathrm{Co}^{16+}$ among other ions. The results adopted by Fuhr et al. (1988) are calculations dating from 1965 (Krueger \& Czyzak) and 1972 
(Tull et al.). Later calculations we have found in the literature are the E2 $g f$-values published by Biémont $\&$ Godefroid (1978) for the sodium sequence up to $Z=26$, and the ones reported by Godefroid et al. (1985) for $3 \mathrm{~s}^{2} \mathrm{~S}-3 \mathrm{~d}^{2} \mathrm{D}$ forbidden transitions of Na-like ions, also up to $Z=26$. In none of these two papers data on $\mathrm{Co}^{16+}$ have been reported. More recently, theoretical electric quadrupole transition probabilities for the Na-like ions $\mathrm{Ba}^{45+}$ through $\mathrm{U}^{81+}$ have been published (Baik et al. 1991). Exploring the web pages, excellent databases containing wavelengths and transition probabilities for allowed and forbidden lines in atoms and ions - see e.g. CHIANTI (http://wwwsolar.nlr.navy.mil/chianti.html) can be found. However, E2 lines of $\mathrm{Co}^{16+}$ are not available. In view of the lack of experimental measurements and the scarce theoretical data available, we have undertaken the calculation of electric quadrupole line strengths and transition probabilities for $n l-n l^{\prime}$ transitions (with $n=$ $3,4,5,6$ and $7 ; l=0,1,2,3$ and 4$)$, and with $\Delta l=0 \pm 2$ for this ion.

For many years we have applied the Quantum Defect Orbital method, both in its non-relativistic (QDO) (Simons 1974; Martín \& Simons 1975, 1976) and its relativistic (RQDO) (Martín \& Karwowski 1991; Karwowski \& Martín 1991) versions, to the calculation of oscillator strengths and photoionization cross sections of a rather large number of atomic species, including several isoelectronic sequences (see, e.g. Martín et al. 1993; Martín et al. 1994; Charro et al. 1996, 1997, 2000; Charro \& Martín 1998), and ions of different degrees of complexity (Biémont et al. 1998; Charro et al. 1999; Charro \& Martín 1999a, 1999b, 2000a, 2000b). The RQDO technique is a simple but reliable analytical method based on a model Hamiltonian. It offers the great advantage that the computational effort is not increased as the atomic system dealt with becomes heavier. It has been found that the RQDO orbitals behave rather well at intermediate and, in particular, large radial distances. These are, in most cases, the regions that contribute more strongly to the transition moment. The convenience of employing exactly solvable model potentials for calculating atomic transition probabilities manifests itself not only from a practical point of view but also because of the involved physical implications.

There is abundant evidence indicating the importance of relativistic effects in highly ionized and in heavy atoms. Relativistic atomic calculations, based on models ranging from simple effective potential approaches to the DiracHartree-Fock ones, have already been performed extensively. Also, the corresponding computer programs are generally available. Nevertheless, it is still of some interest to develop new and simple atomic models, particularly if they are generalizations of the ones commonly used. The relativistic quantum defect orbital (RQDO) method was formulated by Martín \& Karwowski (1991) as a modification of the Schrödinger-like quantum defect orbital (QDO) method (Simons 1974; Martín \& Simons 1975, 1976). The
RQDO approach created a more solid background for its applications in interpreting properties of highly ionised atoms.

In the present work, electric quadrupole line strengths for the multiplets and transition probabilities for the individual lines corresponding to a number of E2 transitions of $\mathrm{Co}^{16+}$ have been computed with the RQDO formalism.

\section{Method of calculation}

The Relativistic Quantum Defect Orbital (RQDO) method has been described in detail in previous papers (Karwowski \& Martín 1991; Martín \& Karwowski 1991; Martín et al. 2000). Therefore, we shall only briefly summarise here its most fundamental aspects.

The relativistic quantum defect orbitals are determined by solving analytically the quasi-relativistic (scalar) second-order Dirac-like equation, obtained after decoupling the radial, two-component Dirac equation, through a non-unitary transformation,

$\left[-\frac{\mathrm{d}^{2}}{\mathrm{~d} r^{2}}+\frac{\Lambda(\Lambda+1)}{r^{2}}-\frac{2 Z_{\text {net }}^{\prime}}{r}\right] \psi_{k}^{\mathrm{RD}}=2 e^{\mathrm{RD}} \psi_{k}^{\mathrm{RD}}$,

with

$Z_{\text {net }}^{\prime}=Z_{\text {net }}\left(1+\alpha^{2} E^{\mathrm{x}}\right)$,

$e^{\mathrm{RD}}=-\frac{\left(Z_{\mathrm{net}}^{\prime}\right)^{2}}{2\left(\eta-\delta^{\prime}\right)^{2}}=E^{\mathrm{x}} \frac{\left(1+\alpha^{2} E^{\mathrm{x}} / 2\right)}{\left(1+\alpha^{2} E^{\mathrm{x}}\right)^{2}}$,

and,

$\Lambda=s-1-\delta^{\prime}+c$,

when $j=l-1 / 2$, or

$\Lambda=-s-\delta^{\prime}+c$

when $j=l+1 / 2$, with $s$ being

$s=k\left(1-\alpha^{2} Z^{2} / k^{2}\right)^{1 / 2}$

and $k$ being the relativistic quantum number in Dirac's notation:

$$
\left.\begin{array}{l}
k=\mp(j+1 / 2) \\
\text { for } j=l \pm 1 / 2
\end{array}\right\} .
$$

Here $n$ and $l$ are the principal and orbital angular momentum quantum numbers and $\eta$ is the relativistic principal quantum number, related with $n$ as follows

$\eta=n-|k|+|s|$

$\delta^{\prime}$ is the relativistic quantum defect, and $c$ is an integer chosen to ensure the normalization of the wavefunction and its correct nodal structure; $Z_{\text {net }}^{\prime}$ is the scaled nuclear charge acting on the valence electrons at large radial distances; $E^{\mathrm{x}}$ is the experimentally measured energy, and $\alpha$ is the fine structure constant. Atomic units are used throughout. Since the effective Hamiltonian in Eq. (2.1) includes a screening term, the quantum defect orbitals are 
approximately valid in the core region of space. Core polarization effects are implicitly included in the calculations as they are accounted for in the $\Lambda$ parameter of the model Hamiltonian. On the other hand, given the one-electron nature of the RQDO methodology, it can be expected to lead to better results in highly excited states, where the active electron interacts less with the core and other valence electrons, than in low-lying energy states. The relativistic quantum defect orbitals lead to closed-form analytical expressions for the transition integrals. This allows us to calculate transition probabilities and oscillator strengths by simple algebra and with high computational efficiency.

The RQDO methodology supplies one-electron radial wavefunctions, characterised by the $n, l$ and $j$ quantum numbers (Martín \& Karwowski 1991), that are used in the transition matrix elements for the initial and final states of the active electron. These correspond to levels of a given $L S J$ symetry in many-electron atoms. We take care of the presence of the remaining electrons by including the appropriate angular factors in the line strengths. Thus, the electric quadrupole line strength for a transition between two states within the $L S J$-coupling, is given by the equation

$$
\begin{aligned}
S_{\mathrm{E} 2}\left(n l j, n^{\prime} l^{\prime} j^{\prime}\right)= & \frac{2}{3}(2 J+1)\left(2 J^{\prime}+1\right) \\
& \times W\left(S J L^{\prime} 2, L J^{\prime}\right)^{2} R_{\mathrm{mult}}^{2}\left(\alpha L, \alpha^{\prime} L^{\prime}\right) \\
& \times\left\langle L\left\|C^{(2)}\right\| L^{\prime}\right\rangle^{2}\left|\left\langle R_{n l j}|Q(r)| R_{n^{\prime} l^{\prime} j^{\prime}}\right\rangle\right|^{2}
\end{aligned}
$$

where $Q(r)$ is the quadrupole transition operator, $r^{2}$, $R_{\text {mult }}$ is the multiplet factor, $W\left(S J L^{\prime} 2, L J^{\prime}\right)$ is the Racah coefficient, and $\left\langle L\left\|C^{(2)}\right\| L^{\prime}\right\rangle$ is the pertinent reduced matrix element, which can be evaluated using $6 j$ - and $3 j$ symbols, respectively.

The total line strength of the multiplet is equal to the sum of the line strengths of all the multiplet lines:

$$
S\left(\gamma L, \gamma^{\prime} L^{\prime}\right)=\sum S\left(\gamma J, \gamma^{\prime} J^{\prime}\right)
$$

The relationships between the line strength $S$ (in atomic units, $e^{2} a_{\mathrm{o}}^{4}$ ), the oscillator strength $f$ (dimensionless), and the transition probability $A$ (in s ${ }^{-1}$ ) are given by

$$
\begin{gathered}
g^{\prime} A=\left(8 \pi^{2} \hbar \alpha / m \lambda^{2}\right) g f=\left(6.6703 \times 10^{15} / \lambda^{2}\right) g f \\
g^{\prime} A_{\mathrm{E} 2}=\left(32 \pi^{5} \alpha c a_{\mathrm{o}}^{4} / 15 \lambda^{5}\right) S_{\mathrm{E} 2} \\
=\left(1.11995 \times 10^{18} / \lambda^{5}\right) S_{\mathrm{E} 2}
\end{gathered}
$$

where $\lambda$ is the transition wavelength (in $\AA$ ), and $g^{\prime}$ and $g$ are the degeneracies of the upper and lower states, respectively.

\begin{tabular}{|c|c|c|c|}
\hline Even State & $\delta^{\prime}$ & Odd State & $\delta^{\prime}$ \\
\hline $3 \mathrm{~s}{ }^{2} \mathrm{~S}_{1 / 2}$ & 0.3200 & $3 p{ }^{2} \mathrm{P}_{1 / 2}$ & 0.2256 \\
\hline $4 \mathrm{~s}^{2} \mathrm{~S}_{1 / 2}$ & 0.3114 & $3 p{ }^{2} \mathrm{P}_{3 / 2}$ & 0.2170 \\
\hline $5 \mathrm{~s}{ }^{2} \mathrm{~S}_{1 / 2}$ & 0.3096 & $4 p{ }^{2} \mathrm{P}_{1 / 2}$ & 0.2151 \\
\hline $3 \mathrm{~d}{ }^{2} \mathrm{D}_{3 / 2}$ & $0.6964(-1)$ & $4 p{ }^{2} \mathrm{P}_{3 / 2}$ & 0.2064 \\
\hline $3 \mathrm{~d}^{2} \mathrm{D}_{5 / 2}$ & $0.6817(-1)$ & $5 \mathrm{p}{ }^{2} \mathrm{P}_{1 / 2}$ & 0.2115 \\
\hline $4 \mathrm{~d}^{2} \mathrm{D}_{3 / 2}$ & $0.7287(-1)$ & $5 p{ }^{2} \mathrm{P}_{3 / 2}$ & 0.2034 \\
\hline $4 \mathrm{~d}^{2} \mathrm{D}_{5 / 2}$ & $0.7137(-1)$ & $6 p{ }^{2} \mathrm{P}_{1 / 2}$ & 0.1996 \\
\hline $5 \mathrm{~d}^{2} \mathrm{D}_{3 / 2}$ & $0.7408(-1)$ & $6 p{ }^{2} \mathrm{P}_{3 / 2}$ & 0.2085 \\
\hline $5 \mathrm{~d}^{2} \mathrm{D}_{5 / 2}$ & $0.7249(-1)$ & $4 \mathrm{f}^{2} \mathrm{~F}_{5 / 2}$ & $0.8187(-2)$ \\
\hline $6 \mathrm{~d}{ }^{2} \mathrm{D}_{3 / 2}$ & $0.7446(-1)$ & $4 f{ }^{2} \mathrm{~F}_{7 / 2}$ & $0.7534(-2)$ \\
\hline $6 \mathrm{~d}^{2} \mathrm{D}_{5 / 2}$ & $0.7288(-1)$ & $5 \mathrm{f}^{2} \mathrm{~F}_{5 / 2}$ & $0.1049(-1)$ \\
\hline $7 \mathrm{~d}^{2} \mathrm{D}_{3 / 2}$ & $0.7408(-1)$ & $5 f{ }^{2} \mathrm{~F}_{7 / 2}$ & $0.1029(-1)$ \\
\hline $7 \mathrm{~d}^{2} \mathrm{D}_{5 / 2}$ & $0.7161(-1)$ & $6 \mathrm{f}^{2} \mathrm{~F}_{5 / 2}$ & $0.1160(-1)$ \\
\hline $5 \mathrm{~g}{ }^{2} \mathrm{G}_{7 / 2}$ & $0.2432(-2)$ & $6 \mathrm{f}^{2} \mathrm{~F}_{7 / 2}$ & $0.1150(-1)$ \\
\hline $5 \mathrm{~g}{ }^{2} \mathrm{G}_{9 / 2}$ & $0.1841(-2)$ & $7 \mathrm{f}^{2} \mathrm{~F}_{5 / 2}$ & $0.1441(-1)$ \\
\hline - & & $7 f{ }^{2} \mathrm{~F}_{7 / 2}$ & $0.1474(-1)$ \\
\hline
\end{tabular}

\section{Numerical results}

The energy data required as input in our procedure (even though when these data are not available, extrapolations can be made on the basis of the regular behaviour of the
Table 1. Relativistic quantum defects for even and odd states of $\mathrm{Co}^{16+}$.

In this and the remaining tables, $A(B)$ denotes $A \times 10^{(B)}$.

quantum defects in an unperturbed spectral series and, sometimes, along an isoelectronic sequence) have been taken from the critical compilation by Kelly (1987). The quantum defects we have extracted from these data are collected in Table 1.

According to Eqs. (2.4) and (2.5), a value for the parameter $c$ has to be chosen, with a narrow range, in order to best describe the RQDO wavefunctions. For all the transitions studied here, the interger $c$ has been chosen to be zero for both, initial and final states, which leads to RQDO wavefunctions that possess the same number of radial nodes as their hydrogenic counterparts.

Before performing the transition probability calculations, we have tested the stability of our wavefunctions with respect to variations in the energy data used as input. The ionization energy (I.E.) of $\mathrm{Co}^{16+}$, as well as the energy of the levels involved in the transitions, are the empirical parameters we need for extracting the quantum defects employed in building the RQDO wavefunctions. The adopted value for the I.E. is $4408500 \pm 300 \mathrm{~cm}^{-1}$, as compiled by Kelly (1987) and formerly derived by Edlén (1978) from core polarization theory applied to the $n \mathrm{f}$ series of the sodium-like ions. Different degrees of uncertainty were estimated for the energy levels of $\mathrm{Co}^{16+}$, the largest corresponding to the $5 \mathrm{~s}{ }^{2} \mathrm{~S}_{1 / 2}$ level, $2967700 \pm$ $3000 \mathrm{~cm}^{-1}$ as earlier reported by Fawcett et al. (1972).

In order to sample how the above uncertainties influence the stability of the RQDO wavefunctions, we have 
Table 2. RQDO line strengths for the $5 \mathrm{~s} \rightarrow n^{\prime} \mathrm{d}$ fine-structure and multiplet transitions.

\begin{tabular}{||c|l|l|l|l||}
\hline Transition & RQDO & ${ }^{2} \mathrm{~S}_{1 / 2}-{ }^{2} \mathrm{D}_{3 / 2}$ & ${ }^{2} \mathrm{~S}_{1 / 2}-{ }^{2} \mathrm{D}_{5 / 2}$ & ${ }^{2} \mathrm{~S}-{ }^{2} \mathrm{D}$ \\
\hline $5 \mathrm{~s} \rightarrow 5 \mathrm{~d}$ & $\mathrm{a}$ & $0.996(+1)$ & $0.150(+2)$ & $0.249(+2)$ \\
& $\mathrm{b}$ & $0.991(+1)$ & $0.149(+2)$ & $0.248(+2)$ \\
& $\mathrm{c}$ & $0.100(+2)$ & $0.150(+2)$ & $0.251(+2)$ \\
$5 \mathrm{~s} \rightarrow 6 \mathrm{~d}$ & $\mathrm{a}$ & $0.190(+1)$ & $0.282(+1)$ & $0.472(+1)$ \\
& $\mathrm{b}$ & $0.184(+1)$ & $0.274(+1)$ & $0.457(+1)$ \\
& $\mathrm{c}$ & $0.196(+1)$ & $0.291(+1)$ & $0.487(+1)$ \\
$5 \mathrm{~s} \rightarrow 7 \mathrm{~d}$ & $\mathrm{a}$ & 0.252 & 0.377 & 0.629 \\
& $\mathrm{~b}$ & 0.248 & 0.371 & 0.619 \\
& $\mathrm{c}$ & 0.256 & 0.383 & 0.639 \\
\hline
\end{tabular}

a: Calculations using I.E. $=4408500 \mathrm{~cm}^{-1}$ and $E_{5 \mathrm{~s}}=2967700 \mathrm{~cm}^{-1}$;

b: Using I.E. $=4408800 \mathrm{~cm}^{-1}$ and $E_{5 \mathrm{~s}}=2964700 \mathrm{~cm}^{-1}$;

c: With I.E. $=4408200 \mathrm{~cm}^{-1}$ and $E_{5 \mathrm{~s}}=2970700 \mathrm{~cm}^{-1}$.

performed three separate calculations of fine-structure and multiplet line strengths for transitions that involve the $5 \mathrm{~s}{ }^{2} \mathrm{~S}_{1 / 2}$ level. The results are collected in Table 2. For each $5 \mathrm{~s}{ }^{2} \mathrm{~S}_{1 / 2}-n d{ }^{2} \mathrm{D}_{3 / 2,5 / 2}$ transitions we have employed three different values of the I.E. and the $5 \mathrm{~s}{ }^{2} \mathrm{~S}_{1 / 2}$ energy. First, $4408500 \mathrm{~cm}^{-1}$ and $2967700 \mathrm{~cm}^{-1}$, respectively were taken. Then the error limits were added in such a way that the largest possible difference between the I.E. and the $5 \mathrm{~s}^{2} \mathrm{~S}_{1 / 2}$ energy was obtained, i.e., $4408800 \mathrm{~cm}^{-1}$ and $2964700 \mathrm{~cm}^{-1}$, respectively. And last, the error bounds were added to these quantities in the opposite sense, so that we obtained $4408200 \mathrm{~cm}^{-1}$ for the I.E. and $2970700 \mathrm{~cm}^{-1}$ for the binding energy of the $5 \mathrm{~s}{ }^{2} \mathrm{~S}_{1 / 2}$ level. Little differences are observed in the line strengths calculated for each of the $5 \mathrm{~s}^{2} \mathrm{~S}_{1 / 2}-n \mathrm{~d}{ }^{2} \mathrm{D}_{3 / 2,5 / 2}$ transitions with the three sets of data, which have been grouped as three entries in Table 2. The discrepancies are less than $1 \%$ for the $5 \mathrm{~s}-5 \mathrm{~d}$ transitions, about $3 \%$ for the $5 \mathrm{~s}-6 \mathrm{~d}$ transitions, and $1.6 \%$ for the $5 \mathrm{~s}-7 \mathrm{~d}$ line strengths.

We expect to have assessed the stability of the RQDO wavefunctions as regards some variations in the energy data employed in their calculation.

The $S$-values were calculated next in an individual way, and not from the application of the LS-coupling rules within multiplets. We find this useful from a spectroscopic point of view, given their application to spectral analyses in astrophysics and fusion plasma research. For comparison, the multiplet $S$-values for the electric quadrupole transitions (E2) between doublets of Na-like Co are displayed in Table 3. For each transition for which comparative data are available, two sets of $S$-values are given. The RQDO values are collected in the first column. Earlier results are given in the second column. These comprise the multiplet Hartree-Fock strengths reported by Tull et al. (1972).

Results for the $n \mathrm{~s}{ }^{2} \mathrm{~S}-n^{\prime} \mathrm{d}{ }^{2} \mathrm{D}$ transitions with $n=3-5$ and $n^{\prime}=3-7$ are displayed in Table 3 , where a fairly good agreement between the RQDO $S$-values and the ones by Tull et al. (1972) for most of the doublet-doublet transitions are found. The discrepancies are less than $10 \%$. The same feature is observed for $n \mathrm{p}^{2} \mathrm{P}^{\mathrm{o}}-n^{\prime} \mathrm{p}{ }^{2} \mathrm{P}^{\mathrm{o}}$ transitions with $n=3-5$ and $n^{\prime}=4-6$. Our results agree satisfactorily with the comparative data. In fact, for all the transitions, they differ by less than $5 \%$. The $S$-values for the $n \mathrm{p}^{2} \mathrm{P}^{\mathrm{o}}-n^{\prime} \mathrm{f}^{2} \mathrm{~F}^{\mathrm{o}}$ transitions with $n=3-6$ and $n^{\prime}=4-7$ are also collected in Table 3. Again, a general good accord between the set of RQDO $f$-values and those reported by Tull et al. (1972) is found, the discrepancies being less than $6 \%$, with the only exception of the $3 \mathrm{p}^{2} \mathrm{P}^{\mathrm{o}}-6 \mathrm{f}^{2} \mathrm{~F}^{\mathrm{o}}$ and $3 \mathrm{p}^{2} \mathrm{P}^{\mathrm{o}}-7 \mathrm{f}^{2} \mathrm{~F}^{\mathrm{o}}$ multiplets, where they amount to about $17 \%$ and $36 \%$, respectively, and both of these correspond to very small values. The RQDO results for the $n \mathrm{~d}{ }^{2} \mathrm{D}-n^{\prime} \mathrm{d}{ }^{2} \mathrm{D}$ transitions with $n=3-6$ and $n^{\prime}=$ 4-7 have a relative deviation with respect to the HF results (Tull et al. 1972) of less than 5\%. Results for a few $n \mathrm{f}^{2} \mathrm{~F}^{\mathrm{o}}-n^{\prime} \mathrm{p}^{2} \mathrm{P}^{\circ}$ transitions are also included. An inspection of the transitions corresponding to $\Delta l=0$ and $l=3$ reveals excellent similarities in magnitude between the two sets of $S$-values for all of the transitions (the deviations are less than $1 \%$ ).

The RQDO line strengths collected in Table 3 all comply with a feature that is characteristic of the LSJ coupling scheme: the strongest line within a given LS transition array is the one for which $\Delta J=J-J^{\prime}$ coincides with $\Delta L=$ $L^{\prime}-L$, in particular when either $J$ or $J^{\prime}$ is the maximum $J$-value in the array.

A general view of the quality of our results is shown in Fig. 1, where the multiplet line strengths obtained in the present calculations are compared with the theoretical results reported by Tull et al. (1972). The RQDO/HF $S$-values ratios are plotted on a logarithmic scale versus $\log S_{\mathrm{RQDO}}$. It appears that only two of the ratios, which correspond to the $3 \mathrm{p}^{2} \mathrm{P}^{\mathrm{o}}-6 \mathrm{f}{ }^{2} \mathrm{~F}^{\mathrm{o}}$ and $3 \mathrm{p}^{2} \mathrm{P}^{\mathrm{o}}-7 \mathrm{f}^{2} \mathrm{~F}^{\mathrm{o}}$ transitions, are deviating by more than 0.05 , while the rest 
Table 3. Comparative multiplet line strengths a) RQDO; b) Tull et al. (1972).

\begin{tabular}{|c|c|c|}
\hline Transition & This work $^{\mathrm{a}}$ & Previous data \\
\hline $3 \mathrm{~s} \rightarrow 3 \mathrm{~d}$ & 0.236 & 0.227 \\
\hline $3 \mathrm{~s} \rightarrow 4 \mathrm{~d}$ & 0.153 & 0.169 \\
\hline $3 \mathrm{~s} \rightarrow 5 \mathrm{~d}$ & $0.210(-1)$ & $0.220(-1)$ \\
\hline $3 \mathrm{~s} \rightarrow 6 \mathrm{~d}$ & $0.646(-2)$ & $0.661(-2)$ \\
\hline $3 \mathrm{~s} \rightarrow 7 \mathrm{~d}$ & $0.285(-2)$ & $0.268(-2)$ \\
\hline $4 \mathrm{~s} \rightarrow 4 \mathrm{~d}$ & $0.353(+1)$ & $0.351(+1)$ \\
\hline $4 \mathrm{~s} \rightarrow 5 \mathrm{~d}$ & $0.105(+1)$ & $0.115(+1)$ \\
\hline $4 \mathrm{~s} \rightarrow 6 \mathrm{~d}$ & 0.143 & 0.151 \\
\hline $4 \mathrm{~s} \rightarrow 7 \mathrm{~d}$ & $0.436(-1)$ & $0.450(-1)$ \\
\hline $5 \mathrm{~s} \rightarrow 5 \mathrm{~d}$ & $0.249(+2)$ & $0.250(+2)$ \\
\hline $5 \mathrm{~s} \rightarrow 6 \mathrm{~d}$ & $0.472(+1)$ & $0.521(+1)$ \\
\hline $5 \mathrm{~s} \rightarrow 7 \mathrm{~d}$ & 0.629 & 0.667 \\
\hline $3 p \rightarrow 4 p$ & 0.148 & 0.145 \\
\hline $3 p \rightarrow 5 p$ & $0.133(-1)$ & $0.129(-1)$ \\
\hline $3 p \rightarrow 6 p$ & $0.375(-2)$ & $0.360(-2)$ \\
\hline $4 p \rightarrow 5 p$ & $0.145(+1)$ & $0.143(+1)$ \\
\hline $4 p \rightarrow 6 p$ & 0.113 & 0.111 \\
\hline $5 \mathrm{p} \rightarrow 6 \mathrm{p}$ & $0.818(+1)$ & $0.822(+1)$ \\
\hline $3 p \rightarrow 4 f$ & 0.776 & 0.736 \\
\hline $3 p \rightarrow 5 f$ & $0.260(-1)$ & $0.273(-1)$ \\
\hline $3 p \rightarrow 6 f$ & $0.273(-2)$ & $0.330(-2)$ \\
\hline $3 p \rightarrow 7 f$ & $0.428(-3)$ & $0.666(-3)$ \\
\hline $4 \mathrm{p} \rightarrow 4 \mathrm{f}$ & $0.394(+1)$ & $0.396(+1)$ \\
\hline $4 \mathrm{p} \rightarrow 5 \mathrm{f}$ & $0.529(+1)$ & $0.502(+1)$ \\
\hline $4 \mathrm{p} \rightarrow 6 \mathrm{f}$ & 0.369 & 0.361 \\
\hline $4 \mathrm{p} \rightarrow 7 \mathrm{f}$ & $0.719(-1)$ & $0.734(-1)$ \\
\hline $5 p \rightarrow 5 f$ & $0.377(+2)$ & $0.378(+2)$ \\
\hline $5 p \rightarrow 6 f$ & $0.225(+2)$ & $0.216(+2)$ \\
\hline $5 \mathrm{p} \rightarrow 7 \mathrm{f}$ & $0.190(+1)$ & $0.185(+1)$ \\
\hline $6 \mathrm{p} \rightarrow 6 \mathrm{f}$ & $0.199(+3)$ & $0.199(+3)$ \\
\hline $6 \mathrm{p} \rightarrow 7 \mathrm{f}$ & $0.754(+2)$ & $0.719(+2)$ \\
\hline $3 \mathrm{~d} \rightarrow 4 \mathrm{~d}$ & 0.171 & 0.164 \\
\hline $3 \mathrm{~d} \rightarrow 5 \mathrm{~d}$ & $0.123(-1)$ & $0.118(-1)$ \\
\hline $3 \mathrm{~d} \rightarrow 6 \mathrm{~d}$ & $0.310(-2)$ & $0.296(-2)$ \\
\hline $3 \mathrm{~d} \rightarrow 7 \mathrm{~d}$ & $0.125(-2)$ & $0.119(-2)$ \\
\hline $4 \mathrm{~d} \rightarrow 5 \mathrm{~d}$ & $0.184(+1)$ & $0.181(+1)$ \\
\hline $4 d \rightarrow 6 \mathrm{~d}$ & 0.127 & 0.125 \\
\hline $4 d \rightarrow 7 d$ & $0.308(-1)$ & $0.300(-1)$ \\
\hline $5 \mathrm{~d} \rightarrow 6 \mathrm{~d}$ & $0.107(+2)$ & $0.106(+2)$ \\
\hline $5 \mathrm{~d} \rightarrow 7 \mathrm{~d}$ & 0.702 & 0.693 \\
\hline $6 \mathrm{~d} \rightarrow 7 \mathrm{~d}$ & $0.440(+2)$ & $0.440(+2)$ \\
\hline $4 \mathrm{f} \rightarrow 5 \mathrm{p}$ & 0.612 & 0.647 \\
\hline $4 \mathrm{f} \rightarrow 6 \mathrm{p}$ & $0.259(-1)$ & $0.276(-1)$ \\
\hline $5 \mathrm{f} \rightarrow 6 \mathrm{p}$ & $0.652(+1)$ & $0.685(+1)$ \\
\hline $4 f \rightarrow 5 f$ & $0.166(+1)$ & $0.165(+1)$ \\
\hline $4 \mathrm{f} \rightarrow 6 \mathrm{f}$ & $0.931(-1)$ & $0.930(-1)$ \\
\hline $4 \mathrm{f} \rightarrow 7 \mathrm{f}$ & $0.200(-1)$ & $0.201(-1)$ \\
\hline $5 f \rightarrow 6 f$ & $0.120(+2)$ & $0.120(+2)$ \\
\hline $5 f \rightarrow 7 f$ & 0.697 & 0.700 \\
\hline $6 f \rightarrow 7 f$ & $0.542(+2)$ & $0.539(+2)$ \\
\hline
\end{tabular}

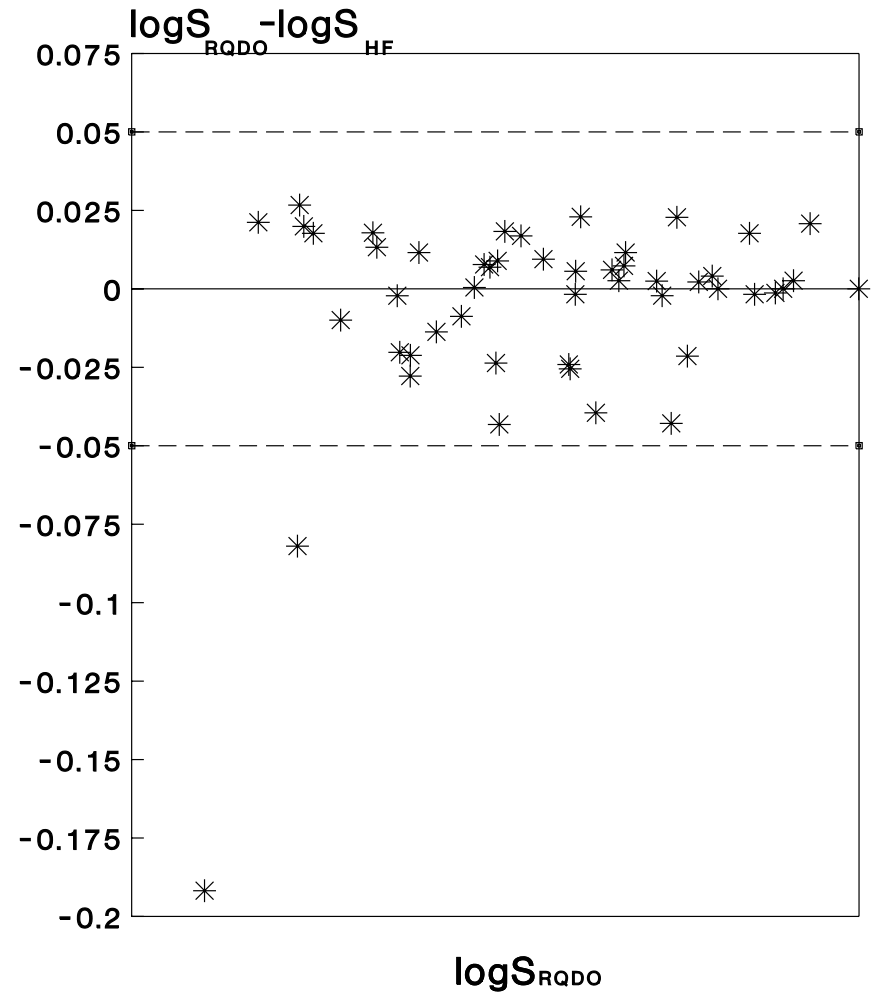

Fig. 1. Comparison of the RQDO E2 multiplet $S$-values for Nalike Co XVII with the results of Tull et al. (1972). Logarithms of the ratios of line strengths $S_{\mathrm{RQDO}} / S_{\mathrm{HF}}$ are plotted vs. $\log S_{\mathrm{RQDO}}$.

of the results are very close to the ratio of 1.00. These discrepancies were already mentioned above. It is our belief that the RQDO data are probably more accurate than the calculations by Tull et al. (1972), given that in the excited states a central field model can be more realistic than in transitions involving lower excited states.

Finally, we collect in Table 4 the transition probabilities for the fine structure lines of the multiplet transition arrays previously displayed in Table 3 , together with some new data for a few transitions involving the $5 \mathrm{~g}{ }^{2} \mathrm{G}_{7 / 2,9 / 2}$ levels. No calculations or measurements are, to our knowledge, available in the literature. In order to facilitate the use of the table to spectroscopists, the spectral lines have been ordered by decreasig wavelength.

\section{Regularities along the spectral series}

The dependence of the hydrogen radial function $R_{n l j}$ with the principal quantum number $n$ can be written as (Berkowitz 1979)

$R_{n l j} \propto \frac{1}{n^{l}}\left[\frac{(n+l)(n+l-1)(n+l-2) \ldots(n-l)}{n^{4}}\right]^{1 / 2}$ 
The number of terms, whose average value is $n$, included into the numerator of the quantity in brackets is $(2 l+1)$, thus

$R_{n l j} \propto \frac{1}{n^{l}}\left[\frac{n^{2 l+1}}{n^{4}}\right]^{1 / 2}=\frac{1}{n^{3 / 2}}$.

The line strength is proportional to the square of the radial transition integral. The variation of the $S$-value for transitions along an unperturbed spectral series is, thus, only dependent on the radial function for the upper state, $R_{n^{\prime} l^{\prime} j^{\prime}}$ :

$S \propto R_{n^{\prime} l^{\prime} j^{\prime}}^{2} \propto \frac{1}{n^{\prime 3}}$.

Thus, for all spectral series of hydrogen or hydrogenlike species, the square of the radial integral diminishes as $n^{-3}$ (Martin \& Wiese 1996 and references therein). When one deals with non-hydrogenic systems, $n^{\prime}$ should be replaced by the effective principal quantum number (Smith et al. 1973),

$n^{*}=n-\delta^{\prime}$,

and the variation of the line strength with $n^{\prime *}$ along a spectral series is such that for sufficiently high $n^{\prime *}$, when the behaviour becomes hydrogenic, satisfies the following expression (Martin \& Wiese 1996):

$S\left(n l j, n^{\prime} l^{\prime} j^{\prime}\right) \propto\left(n^{\prime *}\right)^{-3}$.

It is then deduced that by plotting the $S$-values multiplied by $\left(n^{\prime *}\right)^{3}$ against $\left(n^{* *}\right)$, a constant value will be reached at a given $\left(n^{\prime *}\right)$, if, as remarked above, no configuration mixing is present in the spectral series under study.

In Figs. 2 and 3, the RQDO fine-structure line strengths multiplied by the third power of the effective quantum number of the final state are plotted against $n^{\prime *}$, for several of the spectral series object of the present work. From these figures it may be inferred that the trend followed by our data is such that a constant value will be reached as soon as the upper state is sufficiently excited to acquire a near-hydrogenic character. An anomalous trend is, however, observed in the $4 \mathrm{p}^{2} \mathrm{P}_{1 / 2}-4 \mathrm{f}{ }^{2} \mathrm{~F}_{5 / 2}$ spectral serie, plotted in Fig. 3. This shows an unexpectedly low value on the $Y$ coordinate, which we may interpreted as originated by configuration mixing experienced by the $4 \mathrm{f}_{5 / 2}$ level of $\mathrm{Co}^{16+}$.

\section{Conclusions}

The RQDO formalism has been used to study E2 transitions in $\mathrm{Co}^{16+}$. A good agreement between the RQDO results and the $S$-values available in the literature is observed. The stability of the RQDO wavefunctions with respect to the energy data required as input in our technique has been succesfully tested. Line strengths for several finestructure and multiplet transitions have been calculated, to our knowledge, for the first time.

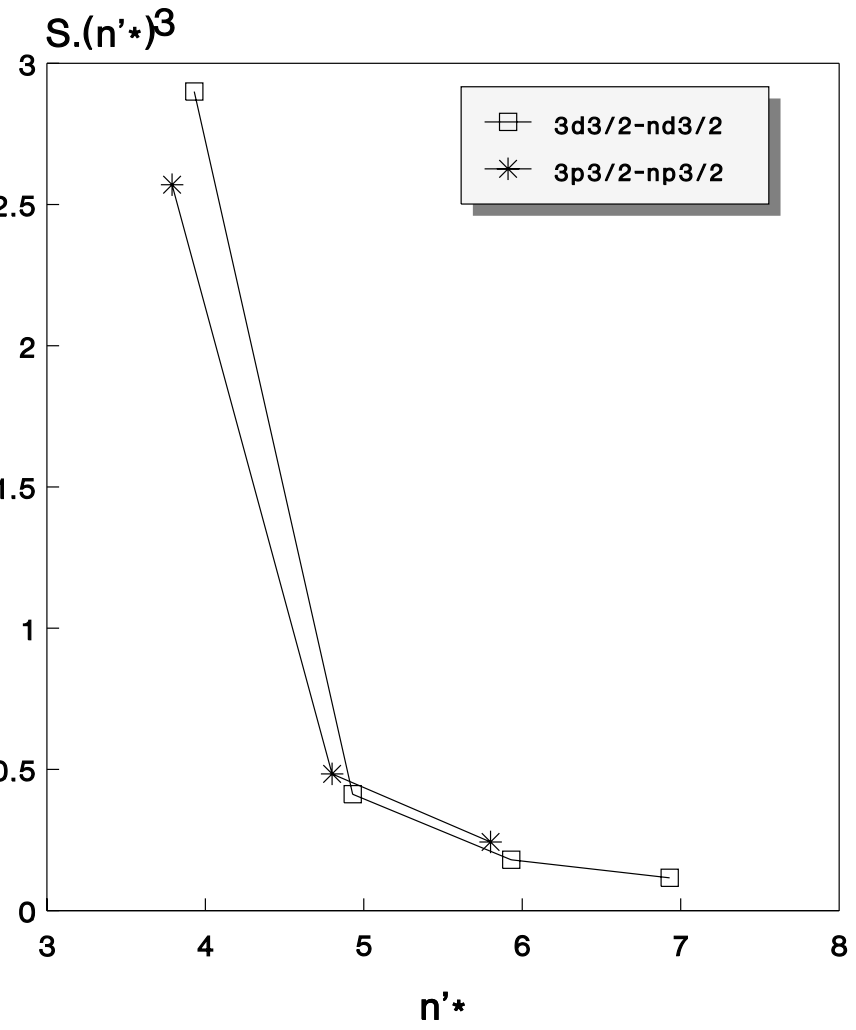

Fig. 2. Regularities in the line strength along the $3 \mathrm{~d}^{2} \mathrm{D}_{3 / 2}-$ $n \mathrm{~d}{ }^{2} \mathrm{D}_{3 / 2}$ and $3 \mathrm{p}^{2} \mathrm{P}_{3 / 2}-n \mathrm{p}{ }^{2} \mathrm{P}_{3 / 2}$ spectral series.

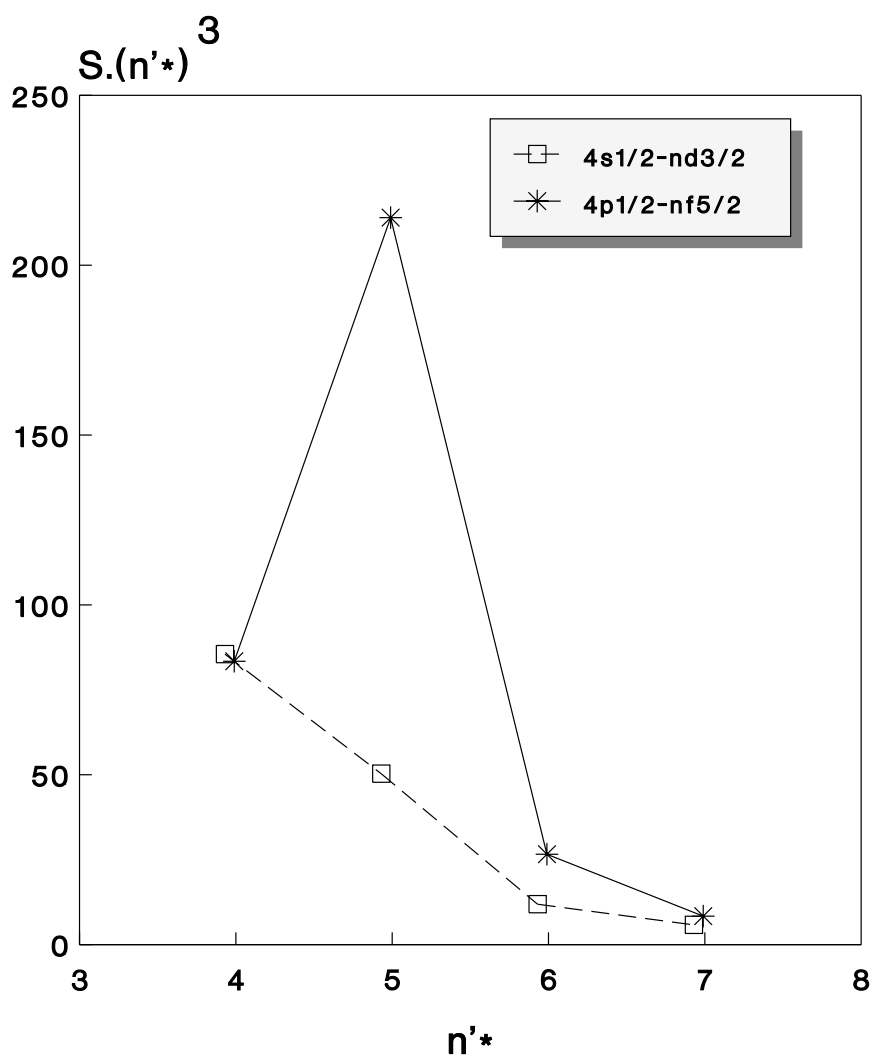

Fig. 3. Regularities in the line strength along the $4 \mathrm{~s}^{2} \mathrm{~S}_{1 / 2}-$ $n \mathrm{~d}{ }^{2} \mathrm{D}_{3 / 2}$ and $4 \mathrm{p}^{2} \mathrm{P}_{1 / 2}-n \mathrm{f}^{2} \mathrm{~F}_{5 / 2}$ spectral series. 
The RQDO procedure has once more proved to be a very useful tool for estimating transition intensities. It is particularly appropriate in the cases where massproduction of data is needed, given its cost-efficiency and reasonable accuracy. It offers also the advantage of being analytic and its lack of convergence problems from which elaborate ab initio calculations often suffer.

Acknowledgements. This work has been supported by the D. G. E. S. of the Spanish Ministry of Education within Project No. PB97-0399-C03-01.

\section{References}

Baik, D. H., Ohr, Y. G., Kim, et al. 1991, At. Data Nucl. Data Tab., 47,177

Berkowitz, J. 1979, Photoabsortion, Photoionization, and Photoelectron Spectroscopy (Academic Press, New York), 20

Biémont, E., Dutrieux, J. F., Martín, I., \& Quinet, P. 1998, J. Phys. B., 31, 3321

Biémont, E., \& Godefroid, M. 1978, Phys. Scr., 18, 323

Charro, E., López-Ayuso, J. L., \& Martín, I. 1999, J. Phys. B., 32,4555

Charro, E., \& Martín, I. 2000a, ApJS, 126, 127

Charro, E., \& Martín, I. 2000b, ApJS, 126, 551

Charro, E., \& Martín, I. 1999a, A\&A, 343, 634

Charro, E., \& Martín, I. 1999b, ApJ, 513, 997

Charro, E., \& Martín ,I. 1998, A\&AS, 131, 523

Charro, E., Martín, I., \& Lavín, C. 1996, J. Quant. Spectrosc. Radiat. Transfer, 56, 241

Charro, E., Martín, I., \& Lavín, C. 1997, A\&AS, 124, 397
Charro, E., Martín, I., \& Serna, M. A. 2000, J. Phys. B, 33, 1753

Edlén, B. 1978, Phys. Scr., 17, 565

Fawcett, B. C., Cowan, R. D., \& Hayes, R. W. 1972, J. Phys. B, 5,2143

Feldman, U. 1992, Phys. Scr., 46, 202

Fuhr, J. R., Martin, G. A., \& Wiese, W. L. 1988, J. Phys. Chem. Ref. Data, 17, Suppl. 4

Godefroid, M., Magnusson, C. E., Zetterberg, P. O., \& Joelsson, I. 1985, Phys. Scr., 32, 125

Karwowski, J., \& Martín, I. 1991, Phys. Rev. A, 43, 4832

Kelly, R. L. 1987, J. Phys. Chem. Ref. Data, 16, Suppl. 1

Krueger, T. K., \& Czyzak, S. J. 1965, Mem. R. Astron. Soc., 69, 145

Kurucz, R. L. 1990, Atomic Spectra and Oscillator Strengths for Astrophysics and Fusion Research, ed. J. E. Hansen (North Holland, Amsterdam), 20

Martín, I., \& Karwowski, J. 1991, J. Phys. B, 24, 1538

Martín, I., Karwowski, J., \& Biélinska-Waz, D. 2000, J. Phys. A, 33, 823

Martín, I., Karwowski, J., Diercksen, G. H. F., \& Barrientos, C. 1993, A\&AS, 100, 595

Martín, P., Lavín, C., \& Martín, I. 1994, Z. Phys. D, 30, 279

Martín, I., \& Simons, G. 1975, J. Chem. Phys., 62, 4799

Martín, I., Simons, G. 1976, Mol. Phys., 32, 1017

Martin, W. C., \& Wiese, W. L. 1996, in Atomic, Molecular, and Optical Physics Handbook, American Institut of Physics, ed. G.W.F. Drake (Woodbury, New York), 135

Simons, G. 1974, J. Chem. Phys., 60, 645

Smith, M. W., Martin, G. A., \& Wiese, W. L. 1973, Nucl. Inst. Meth., 110, 219

Tull, C. E., Jackson, M., McEachran, P., \& Cohen, M. 1972, J. Quant. Spectrosc. Radiat. Transfer, 12, 893 